\title{
ELECTRONIC STRUCTURE OF THE $\mathrm{CdTe}(100)-(1 \times 1)$ SURFACE
}

\author{
K.-U. Gawlik, J. Brügmann, S. Harm, C. Janowitz, R. Manzke, \\ M. SкIBowski
}

Institut für Experimentalphysik, Universität Kiel, D-2300 Kiel 1, Germany

$$
\text { C.-H. Solterbeck, W. Schattke }
$$

In.stitut für Theoretische Physik, Universität Kiel, D-2300 Kiel 1, Germany

\author{
AND B.A. ORLOWSKI \\ Institute of Physics, Polish Academy of Sciences \\ Al. Lotników 32/46, 02-668 Warszawa, Poland
}

Unreconstructed CdTe(100) surface prepared by ion bombardment and annealing is investigated by angle-resolved photoemission. The experimental band structure $E\left(k_{\|}\right)$is determined along high-symmetry lines of the surface Brillouin zone by measuring energy-distribution curves of photoelectrons. Different criteria were applied to separate surface and bulk related spectral features, e.g. calculating the position of bulk-derived emissions in the frame of the free-electron final state approximation assuming $k$ conservation. In this way, most dispersing features could be explained. All remaining features were compared with theoretical surface band structures for different polar surface terminations which were calculated within a layer doubling procedure on the basis of an EHT-fit to the bulk band structure. The investigated $\mathrm{CdTe}(100)-(1 \times 1)$ surface could be identified as Cd terminated. Two surface bands were observed, one located above the valence-band edge and the second in the open pocket of the projected bulk band structure along the $\bar{T} \bar{K}$ direction. At $4.6 \mathrm{eV}$ binding energy an additional weakly dispersing band was found, which contains mixed surface and bulk character. The high density of bulk states associated with this edge of the heteropolar gap is also expected to contribute to this feature.

PACS numbers: 78.55.Et, 79.60.Eq 


\section{Introduction}

In recent years, II-VI and III-V semiconductors with their best studied representatives CdTe and GaAs respertively, have gained increasing technological interest, especially concerning the fabrication of optoelectronical devices. It is therefore of great importance to study the electronic bulk and surface structure of these materials in great detail in order to understand the electric properties. The cleavable (110) surface of CdTe has become subject to band structure investigations by means of angle-resolved photoemission spectroscopy (AR.PES) and similarities between surface and bulk properties of CdTe and III--V semiconductor compounds have been demonstrated [1]. Concerning the (100) inysial plane, GaAs reveals a couple of various surface reconstructions [2-4], but in unreconstructed $(1 \times 1)$ surface structure has not been observed. In contrast 1, GaAs, Takahashi and Ebina [5] reported that the CdTe $(100)$ surface posesses $(1 \times 1)$ structure showing up important differences concerning the surface related mechanisms of these two types of semiconductors. The $(1 \times 1)$ structure appeared upon annealing of $\mathrm{CdTe}(100)-(3 \times 1)$ surface and thus seems to be more stable termination. Up to now, the only experimental investigation on the electronic structure of $\mathrm{CdTe}(100)$ has been published by Niles and Höchst [6]. These authors took normil emission ARPES data of $\mathrm{CdTe}(100)-(2 \times 1)$ surface and studied exclusively the bulk electronic properties. In this paper we present the first investigation of the surface electronic structure of $\mathrm{CdTe}(100)-(1 \times 1)$. The detailed experiniental and theoretical analysis explains surface as well as bulk derived electronic emissions in the spectra. In addition, we calculated the surface band structure of $\mathrm{CdTe}(100)-(1 \times 1)$ including relaxation, which allows us to determine the termination of the surface prepared in the experiment.

\section{Experimental}

The angle-resolved photoemission spectra were taken with synchrotron radiation in a photon energy range of $9 \mathrm{eV} \leq h \nu \leq 32 \mathrm{eV}$ supplied from the storage ring DORIS III at HASYLAB in Hamburg, FR Germany, with an energy resolution of $80 \mathrm{meV}$ and with HeI radiation $(h \nu=21.22 \mathrm{eV})$ with tul energy resolution of $144 \mathrm{meV}$. The electrons were detected by means of a spher inal inlalyzer mounted on a two-axes goniometer. The angle resolution was better thin11 $0.5^{\circ}$.

The $\mathrm{CdTe}(100)$ wafers with dimensions of $5 \times 5 \times 0.5111 \mathrm{In}^{3}$ wer? cut from CdTe single crystals, which were grown by the modified Bridgluan method in the Institute of Physics, Polish Academy of Sciences, and oriented by stanclard X-ray diffraction. All samples investigated here were cleaned by $500 \mathrm{eV}$ argout ion bombardment for 15 minutes and then annealed at about $260^{\circ} \mathrm{C}$ for 2 hours. T'he surface structure was characterized by means of low-energy electron diffraction (LEED) and reflection high-energy electron diffraction (RHEED) in an ultrahigh-vacuum (base pressure in the low $10^{-10} \mathrm{mbar}$ range) preparation system. Fron all investigations we arrived at an unreconstructed $\mathrm{CdTe}(100)$ surface by the conditions given above. 


\section{Results and discussion}

Before a detailed discussion of the surface band structure, $E\left(k_{\|}\right)$, the bulk valence band maximum (VBM) $\Gamma_{8}$ should be determined, i.e. via the photon energy at which the emissions from the upperr. ost bulk valence band $\Gamma_{8} X_{7}$ reach maximum kinetic energy. A selection of norın al emission spectra $\left(\vartheta=0^{\circ}\right)$ taken at various photon energies corresponding to $k_{L}-$-wave vectors along the $\Gamma \Delta X$-direction of the bulk Brillouin zone is shown in Fig. 1. The first emission maxima at highest

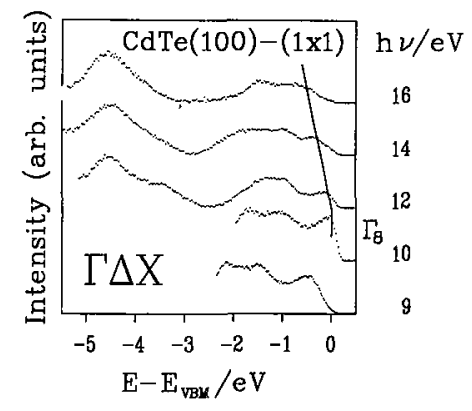

Fig. 1. Energy distribution curves at normal emission at various photon energies $h \nu$. The binding energy is related to the bulk valence band maximum $\Gamma_{8}$ (see text). The emission maxima connected by the line are the emission features of each spectrum with the lowest binding energy which allowed the determination of the valence band maximum $\Gamma_{8}$.

kinetic energy or vice versa at low binding energy of each spectrum are connected by a line. These maxima can be interpreted as direct transitions from the $\Gamma_{8} X_{7}$ valence band into a final state band. One can continuously follow the dispersion of the first structure with decreasing photon energy down to $10 \mathrm{eV}$ moving towards lower binding energy. For slightly lower photon energies direct transitions from $\Gamma_{8}$ would fall into an energy gap of the final states ruling out $\Gamma_{8}$ as the origin of the $0.5 \mathrm{eV}$ peak in the $9 \mathrm{eV}$ spectrum. This situation, i.e. the changes in the spectra measured with photon energies around the onset of transitions from the valence band maximum $\Gamma_{8}$, is discussed in detail in Ref. [6]. For the: annlysis discussed below we took the energy position of the first peak of the 11 ( $V$ spectrum as the reference for the bulk valence band maximum and the bindiın, mergy scale. This leads to an inner potential related to VBM of $\left|E_{0}\right|=4.2 \mathrm{eV}$, w $\left.l_{1} i_{1}\right]_{1}$ is in reasonable agreement with the corresponding theoretical value (see below).

In order to determine the surface band structure $E\left(k_{\|}\right)$wo measured energy-distribution curves for emission angles $v$ along the $\bar{\Gamma} \bar{K}$ direction of the surface Brillouin zone at $h \nu=21.22 \mathrm{eV}$. A selection of spectra covering a large $k_{\|}$range from $\bar{\Gamma}$ to $\bar{K}$ is shown in Fig. 2. The insert shows the surface Brillouin zone including the direction scanned by the energy distribution curves of Fig. 2. Some emission features are connected by full lines labelled $S_{1}, S_{2}, S, S^{\prime}$, and $D S$. These 


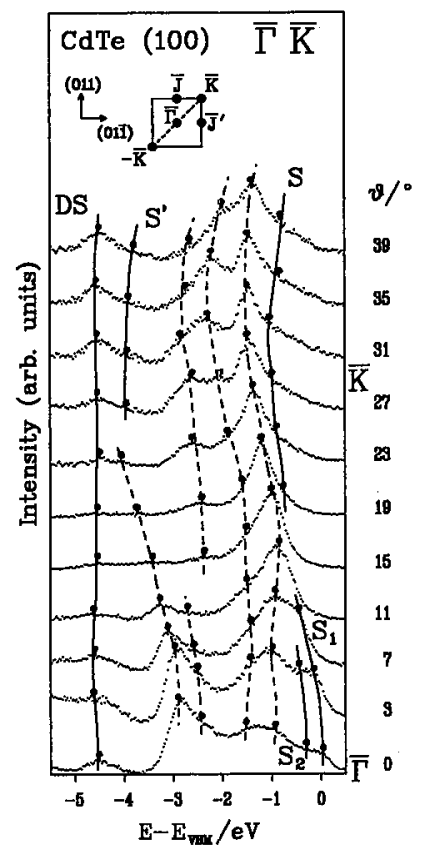

Fig. 2. 1:hotoemission spectra taken parallel to the surface with $21.22 \mathrm{eV}$ photon energy along $\bar{\Gamma} \bar{K}$ as a function of the emission angle $\vartheta$. The insert shows the surface Brillouin zone. The emission maxima were determined by a least squares fit using Gaussian functions. The structures labelled $S_{1}, S_{2}, S, S^{\prime}$ and $D S$ have a dominantly surface character. All other features not labelled are the contributions of transitions from bulk states.

contain dominantly surface character. All other structures not labelled and connected by dashed lines are due to emissions from bulk bands which will be shown below.

In the first step of our analysis we calculated from the spectra series of Fig. 2 the entire experimental band structure $E\left(k_{\|}\right)$taking into consideration all emission maxima due to transitions from bulk and surface states into free-electron-like final states, using the relation $k_{\|}=\sqrt{\left(2 m / \hbar^{2}\right) E_{\text {kin }}} \sin \vartheta$. To separate the surface transitions from bulk transitions we applied in the second step a method developed by Olde et al. [7], which consists in calculating a band structure $E_{\mathrm{tr}}\left(k_{\|}\right)$considering the transition energies of the bulk states at conditions directly comparable with the experiment. The bulk valence band structure taken as the initial states of the transitions, $E_{\mathrm{b}, i}(k)$, is calculated by the empirical tight-binding method (ETBM) [8]. For the final state bands we assume free-electron-like bands described by $E_{\mathrm{b}, f}\left(k_{\|}, k_{\perp}\right)=\left(\hbar^{2} / 2 m\right)\left[\left(k_{\perp}+G_{\perp}\right)^{2}+\left(k_{\|}+G_{\|}\right)^{2}\right]-\left|E_{0}\right|$, where $\left|E_{0}\right|$ is the inner potential related to VBM and which was determined to $\left|E_{0}\right|=4.2 \mathrm{eV}$ from the normal emission spectra. $k_{\|}$and $G_{\|}$are the components parallel to the surface 
of the Bloch wave vector and a reciprocal lattice vector respectively, and $k_{\perp}$ and $G_{\perp}$ - those components perpendicular to the surface. If a bulk band structure $E_{\mathrm{b}, i}(k)$ is given and assuming $k$-conservation, one is then able to calculate for a given photon energy $h \nu$ the emissions from these bands, i.e. direct transitions using $E_{\mathrm{b}, f}(k)-E_{\mathrm{b}, i}(k)=h \nu$. Now we take the part of the $k$-space scanned by our experiment in off-normal emission as a two-dimensional discrete array $\left(k_{\perp, m}, k_{\|, n}\right)$ with the indices $m$ and $n$. For each fixed $k_{\|, n}$ value we vary the $k_{\perp, m}$ component and the valence band index in order to get those values where transitions with a photon energy of $h \nu=21.22 \mathrm{eV}$ into a free-electron-like final state become allowed by the energy and momentum selection rules. This procedure results in wave vectors $\boldsymbol{k}_{\mathrm{tr}}=\left(k_{\perp t r}, k_{\| \mathrm{tr}}\right)$ and energy positions $E_{\mathrm{b}, i}\left(k_{\mathrm{tr}}\right)=E_{\mathrm{b}, f}\left(k_{\mathrm{tr}}\right)-h \nu$ at which transitions occur. These points, $k_{\mathrm{tr}}, E_{\mathrm{b}, i}\left(k_{\mathrm{tr}}\right)$, are projected into the plane with binding energy $E_{\mathrm{b}, i}$ as ordinate and the wave vector component $\boldsymbol{k}_{\| \text {tr }}$ as abscissa. This way we get one-dimensional function $E_{\mathrm{tr}}\left(k_{\|}\right)$which shows, from the theoretical point of view, where emissions from bulk bands should be seen in photoemission. This function can directly be compared with the experimental band structure $E\left(k_{\|}\right)$, with the result that all features not labelled in Fig. 2 (dashed lines) can clearly be attributed to transitions from bulk bands.

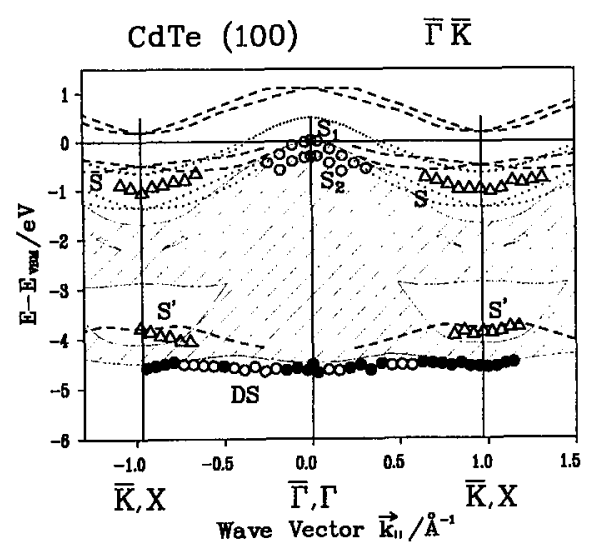

Fig. 3. Surface band structure of $\mathrm{CdTe}(100)-(1 \times 1)$. Together with the projected bulk valence band structure (hatched area) and the calculated surface bands of an ideal surface for $\mathrm{Cd}$ (dashed lines) and Te termination (dotted lines) the experimental surface bands are shown, labelled $S, S_{1}, S_{2}, S^{\prime}$, and $D$ is isee text).

Figure 3 shows the resulting experimental surface band structure for the $\bar{\Gamma} \bar{K}$ direction. Triangles mark clearly identified surface structures, whereas circles represent structures where this evidence is not so strong, mainly because bulk transitions are expected nearby. Full symbols indicate strong, open symbols weaker structures. Additionally, the projected theoretical band structure (hatched area) calculated by ETBM is shown in this picture. Around the Brillouin zone boundary $\bar{K}$ a surface band $S$ is located above the valence band and a surface band labelled $S^{\prime}$ is found in the open pocket of the projected valence band structure, 
which confirms the surface state nature of $S$ and $S^{\prime}$. Besides these, we observe additional bands due to surface resonances labelled $S_{1}, S_{2}$, and $D S$.

The $\mathrm{CdTe}(100)$ surface is polar which means that this surface is either terminated by $\mathrm{Cd}$ or Te. In order to decide the actual experimental termination, we used the surface band structure calculationis performed together with these investigations. There, the parameters of the Exiended-Hückel-Theory (EHT) were determined by a fit of the bulk band structure to various experimental $[9,10]$ and also theoretical band structures. The Hamilton matrix of the semi-infinite crystal is then given by the EHT-parameters and by the calculated overlap matrix in dependence on the surface termination and relaxation. A layer-doubling procedure yields, via the matrix of the Greens function and the density of states, the surface band structure which is shown in Fig. 3 for an ideal Cd and Te terminated surface by dashed and dotted lines respectively. Termination by Te yields theoretically two surface state bands each of them spin-orbit splitted above the valence band edge located near $S$. One pair of these states crosses VBM at about $k_{\|} \approx 0.35 \AA^{-1}$ becoming unoccupied near the $\bar{\Gamma}$-point and predicting the Te terminated $\mathrm{CdTe}(100)$ surface to be metallic. On the other hand, the Cd terminated surface exhibits only one spin-orbit splitted pair below VBM in the energy regime near $S$ which starts to overlap with the bulk band structure near the $\bar{\Gamma}$-point. In the experiment only one surface state band, i.e. $S$, was detected above the projected valence band edge near the $\bar{K}$-point. It cannot be continuously followed through the whole surface Brillouin zone, but it seems to be reasonable to connect $S$ with the surface state $S_{1}$ near $\bar{T}$. The good correspondence between $S$ and $S_{1}$ and the calculated surface band together with the absence of a second pair of states within the gap suggests this surface to be Cd terminated. More evidence arises from the fact that for Te termination, in contrast to $\mathrm{Cd}$ termination, $1, \mathrm{~h}$ : theory predicts no surface state in the open pocket of the projected band structiris where we observed experimentally the surface state band $S^{\prime}$. It should be mentioned here that for the Te termination $S^{\prime}$ is also absent when different relaxations are included in the calculation.

Surface band structure calculations fos: a Cd terminated surface along the high-symmetry directions $\bar{\Gamma} \bar{J}$ and $\bar{\Gamma} \overline{J^{\prime}}$ of the surface Brillouin zone show some contribution to the dispersionless structure $D S$ near $-4.6 \mathrm{eV}$ binding energy which means that this structure may also contain surface character.The high density of bulk states associated with the bulk band edgeat $X_{6}$ is also expected to contribute to this feature. A similar behaviourwas found for the $\mathrm{CdTe}(110)$ surface [1].

\section{Conclusion}

In contrast to $\mathrm{GaAs}$ we were able to prepare an unreconstructed (100) surface for CdTe. We performed a detailed study of the electronic band structure of this $\mathrm{CdTe}(100)-(1 \times 1)$ surface by angle-resolved photoemission spectrosicopy. The bulk valence band maximum $\Gamma_{8}$ was determined by photon energy dependent normal emission energy distribution curves, yielding also the inner potential parameter. From off-normal photoemission the experimental surface band si-icture was derived along the $\bar{\Gamma} \bar{K}$-direction of the surface Brillouin zone. By calculating energy and momentum of the bulk transitions expected for the particular experimental 
conditions it was possible to separate bulk from surface emissions. To decide further whether the investigated surface is terminated by $\mathrm{Cd}$ or Te, we compared the experimentally determined surface bands with the results of a surface band structure calculation considering both possible surface terminations. The examined surface is found to be $\mathrm{Cd}$ terminated with reasonable agreement between experimental and theoretical surface band structure.

\section{Acknowledgernent}

This work is supported by the Bundesministerium für Forscliung und Technologie (project No. 055 FKAAB and 055 FK' $\mathrm{TAB}$ ).

\section{References}

[1] C. Janowitz, L. Kipp, R. Manzke, B.A. Orłowski, Surf. Sci. 231, 25 (1990).

[2] A.Y. Cho, J. Appl. Phys. 42, 2074 (1971).

[3] P. Drathen, W. Ranke, K. Jacobi, Surf. Sci. 77, L162 (1978).

[4] R.Z. Bachrach, R.S. Bauer, P. Chiaradia, G.V. Hansson, J. Vac. Sci. Technol. 18, 797 (1981).

[5] T. 'T'akahashi, A. Ebina, Appl. Surf. Sci 11/12, 268 (1982).

[6] D.W. Niles, H. Höchst, Phys. Rev. B 43, 1.992 (1991).

[7] J. Olde, K.-M. Behrens, H.-P. Barnscheidt, R. Manzke, M. Skibowski, J. Henk, W. Schattke, Phys. Rev. B 44, 6312 (1991).

[8] M. Lehmann, M.Sc. Thesis, Universität Kiel, Kiel 1988.

[9] B.A. Orłowski, Z. Gołacki, C. Janowitz, L. Kipp, R. Manzke, Acta Phys. Pol. A77, 295 (1990).

[10] B.A. Orlowski, C. Janowitz, R. Manzke, E. Janic, Acta Phys. Pol. A 79, 291 (1991). 